\title{
Sign-spatiality in Kata Kolok
}

\section{How a village sign language of Bali inscribes its signing space}

(Radboud University Nijmegen, 2012)

\section{Connie de $\operatorname{Vos}^{1}$}

Max Planck Institute for Psycholinguistics

[Available online at: http://hdl.handle.net/2066/99153]

From its inception, the field of sign linguistics has been pre-occupied with the linguistic significance of sign-spatial mapping, and this thesis builds on this longstanding tradition (see for example Supalla 1978; Poizner, Klima \& Bellugi 1987; Engberg-Pedersen 1993; Emmorey \& Reilly 1995; Taub 2001; Liddell 2003; Perniss 2007). This dissertation presents new data from Kata Kolok, a sign language which emerged in a village community of North Bali and has been the primary means of communication for at least five generations of deaf, native signers (Marsaja 2008; this thesis). Supported by extensive periods of linguistic fieldwork, corpus analysis, as well as targeted, stimulus-based elicitation, it argues that Kata Kolok signers structure their spatial reference system preferentially on the basis of geographical locations, and that they do not utilise the dimensions of the signing space in order to talk about non-spatial concepts.

Part II starts of by summarising what is currently known about Kata Kolok from genetic, anthropological, and sociolinguistic studies that began in the mid1990s. These initial descriptions form the basis for the methodological choices that led to the creation of a Kata Kolok corpus, which currently consists of almost 150 hours of video data. This digital archive includes sections devoted to spontaneous interaction, culturally entrenched narratives, as well as longitudinal recordings of first language acquisition, and deaf-hearing interactions. ${ }^{2}$ The description of research methodology more generally is followed by a grammatical sketch of

1. This research was conducted within the Language \& Cognition department of the Max Planck Intistute for Psycholinguistics.

2. The metadata can be viewed at http://hdl.handle.net/1839/00-0000-0000-0008-CB2B-4@view. 
the structural aspects of Kata Kolok. Along the way, some of the features that are of particular interest in terms of linguistic typology, sign language typology, and cross-modal contact are highlighted. Despite the fact that Kata Kolok has been in intimate contact with spoken Balinese from its incipience, the languages have distinct constituent orders with respect to basic transitive clauses, the placement of negation, as well as numeral-noun and adjective-noun orders. The languages also exhibit marked differences in the degree of lexicalisation in semantic fields such as colour and kinship. From the perspective of sign language typology, one of the most striking findings is the absence of spatial modification of transitive verbs.

Part III begins with presenting a brief overview of the ways in which signspatial mappings have been framed as well as the linguistic evidence that has been offered in support of these analyses. Sign-spatial mapping constitutes a diverse range of phenomena that can and should be analysed at different levels of linguistic structure. Part III coins the term 'sign-spatiality' to delineate the specific type of sign-spatial mapping which this thesis deals with: the ways in which signers systematically employ the degrees of spatial freedom of signs to indicate meanings that are ultimately resolved by the interplay between discourse and grammar. Based on a comparison of sign-spatial structures in Kata Kolok and other sign languages, it is shown that the use of sign-spatiality in Kata Kolok discourse is largely limited to absolute spatial reference. This observation leads to the description of alternative, non-sign-spatial structures that are used in Kata Kolok in the domains of person and time reference. On a par with users of previously documented sign languages, Kata Kolok signers may use list buoys in anaphoric reference, as well as role shift as a means of deferred reference. As in other sign languages, tense is not marked on verbs in Kata Kolok, but the past-future distinction is not usually marked adverbially either leaving temporal reference predominantly based on pragmatic inference. On the whole, Kata Kolok's referential structures are primarily resolved by extra-linguistic elements, and it is hypothesised that this system may be facilitated by the dense social networks within the signing community.

Part IV addresses the nature of sign-spatiality as it relates to spatial Frames of Reference described by Levinson (2003). It raises the issue of sign-spatial significance: due to the fact that signs themselves are essentially spatial, and sign-spatiality is exclusively used as a semiotic strategy in Kata Kolok Figure-Ground constructions, an interpretation in any of the three Frames of Reference is available at any time. This part of the thesis is centred on the analysis of spontaneous narratives, and structured elicitation sets known as the Nijmegen Space Games. The results corroborate previous observations that Kata Kolok signers predominantly commit to an absolute Frame of Reference. This means that Kata Kolok signers foreground absolute spatial relations between elements of a scene being described, and generally background their own view of the scene. In contrast to signers of 
previously described sign languages, Kata Kolok interlocutors do not perform a mental rotation of simultaneous classifiers when interpreting them. The analysis also shows that deaf Kata Kolok signers adopt variable strategies in solving spatialcognition tasks. This finding is taken to indicate that the choice for a particular Frame of Reference in such cases is influenced by additional eco-cultural factors.

Part V examines pointing signs, which have been traditionally analysed according to their sign-spatial reference to locations and associated entities. Corpus analysis of over one thousand points shows that one in six manual signs is a pointing sign in Kata Kolok, and that pointing signs serve a wide variety of functions, including reference to locations, individuals, colours, body parts, and times of day. Across these functions it is found that the sign-spatial properties of pointing signs are rarely the sole determining factor in their interpretation. Instead, I argue that context, the construction in which a pointing sign occurs, and the formal properties of the pointing sign itself, as well as simultaneous non-manual marking on the face, cue the meaning of a pointing sign. The interpretation of pointing signs in Kata Kolok thus takes into account multiple, composite and incremental signals. This section of the thesis also discusses the grammatical nature of Kata Kolok pointing system as reflected by the degree to which they are morphemised (have systematic form-meaning mappings) and syntactically integrated (in term of filling specific syntactic slots in the utterance). While similar patterns are attested in the literature on pointing gestures in both signed and spoken languages, I argue that we need to compare pointing gestures and pointing signs on equal ground, and this means including all aspects of their semiotic ecologies. Preliminary observations indicate that pointing signs and pointing gestures differ in their relative frequency, communicative load, degree of functional diversification, and compulsoriness, because they are integrated into different semiotic ecologies.

While this thesis aims to provide the reader some insights into the sign-spatial phenomena in the language, the data collection and analysis have not systematically targeted the factors that may have led to Kata Kolok's unusual sign-spatial fingerprint. Such factors could include the impact of Balinese co-speech gesture, L2 learner effects caused by the larger group of semi-fluent signers, and possible inter-generational differences as resulting from the language's relatively limited time depth. Although these aspects of Kata Kolok fall beyond the scope of this thesis, I touch upon them by comparing the findings from Kata Kolok to the literature on Balinese gesture, as well as other (village) sign languages and spoken languages in sections pertaining to these issues in the final thesis part in particular. These cross-linguistic comparisons aim to enable the reader to situate the findings from Kata Kolok in the context of sign language typology. I argue that, on a par with spoken language typology, the analysis of sign languages needs to take into account cross-linguistic variation in the domain of spatial language as expressed 
by sign-spatial constructions. These descriptions could ultimately serve a crossmodal typology - the empirically grounded investigation of intramodal and intermodal differences between both natural language modalities - in the domain of space, in particular.

\section{References}

Emmorey, Karen \& Judy Reilly (eds.). 1995. Language, gesture, and space. Hillsdale, NJ: Lawrence Erlbaum.

Engberg-Pedersen, Elisabeth. 1993. Space in Danish Sign Language. The semantics and morphosyntax of the use of space in a visual language. Hamburg: Signum.

Levinson, Steven C. 2003. Space in language and cognition: Explorations in cognitive diversity. Cambridge: Cambridge University Press.

Liddell, Scott K. 2003. Grammar, gesture and meaning in American Sign Language. Cambridge: Cambridge University Press.

Marsaja, I Gede. 2008. Desa Kolok - a deaf village and its sign language in Bali, Indonesia. Nijmegen: Ishara Press.

Perniss, Pamela M. 2007. Space and iconicity in German Sign Language (DGS). PhD dissertation, Radboud University Nijmegen.

Poizner, Howard, Edward Klima \& Ursula Bellugi. 1987. What the hands reveal about the brain. Cambridge, MA: MIT Press.

Supalla, Ted. 1978. Morphology of verbs of motion and location in American Sign Language. In Frank Caccamise (ed.), American Sign Language in a bilingual, bicultural context. Proceedings of the Second National Symposium on Sign Language Research and Teaching, 27-45. Silver Spring, MD: National Association of the Deaf.

Taub, Sarah. 2001. Language from the body: Iconicity and metaphor in American Sign Language. Cambridge: Cambridge University Press.

\section{Table of contents}

\section{Part I: Introduction}

1 When space is special: sign-spatial mapping

1.1 Conventionalisation of space across cultures and languages

1.2 Bengkala and Kata Kolok

1.3 A brief introduction to sign linguistics

1.4 Cross-linguistic variation in sign-spatial mappings

1.4.1 Using the signing space to talk about space

1.4.2 Grammatical person and the signing space

1.4.3 Time and the signing space

1.4.4 Spatial and non-spatial functions of sign-spatial mapping

1.5 Scope and structure of the thesis

Part II: Preliminaries

2 Bengkala and Kata Kolok: a deaf village and its sign language

2.1 Overview 
2.2 Bengkala: a deaf village in the north of Bali

2.2.1 Village life in Bengkala

2.2.2 The Kata Kolok signing community

2.2.3 The social construction of deafness in Bengkala

2.3 Size of the signing community

2.4 Acquisition of Kata Kolok

2.5 Time depth of Kata Kolok

2.6 Bilingualism and endangerment of Kata Kolok

2.7 Previous linguistic analyses of Kata Kolok

2.8 Summary and discussion

3 Research methodology

3.1 Overview

3.2 Ethical aspects with respect to fieldwork

3.2.1 Obtaining consent

3.2.2 Deaf education program

3.2.3 Community involvement

3.3 Linguistic fieldwork

3.4 Creation of the Kata Kolok corpus

3.4.1 Contributors

3.4.2 Metadata

3.4.3 Translations, annotations, and coding

3.4.4 Sub-corpora

3.5 Summary and discussion

4 Structural aspects of Kata Kolok

4.1 Overview

4.2 Manual and non-manual forms

4.2.1 Handshapes

4.2.2 Non-manual signals

4.2.3 Sign language glosses

4.3 The lexicon

4.3.1 Differential lexicalisation in Kata Kolok and Balinese

4.3.2 Limited contact-induced structures

4.3.3 The lexica of Kata Kolok and other village sign languages

4.4 Numeral incorporation

4.5 Classifiers

4.5.1 Entity classifiers

4.5.2 Handle classifiers

4.6 Intensification

4.6.1 Intensification of colour signs

4.6.2 Intensification of pointing signs

4.6.3 Intensification of other signs

4.6.4 The semantics of intensification

4.7 Aspect

4.7.1 Completive aspect

4.7.2 Negative completive aspect

4.7.3 Distributive aspect

4.8 Verb classes 
4.8.1 Plain, agreement and spatial verbs

4.8.2 Transitive constructions in Kata Kolok

4.8.3 Body-anchored verbs

4.8.4 Verb inflection and time depth

4.9 Constituent order

4.9.1 Constituent order in transitive sentences

4.9.2 Position of negation

4.9.3 Noun phrases

4.9.4 Kata Kolok and Balinese constituent orders

4.10 Summary and discussion

Part III: Sign-spatiality at the discourse level

5 Sign-spatiality

5.1 Overview

5.2 Sign-spatial mapping: various levels of structural organisation

5.3 Sign-spatiality: deixis and anaphora

5.4 The semiotics of sign-spatiality

5.5 Summary and discussion

6 Spatial deixis in Kata Kolok

6.1 Overview

6.2 The neutral and the extended signing space

6.3 Sign-spatial directions of pointing signs

6.4 Sign-spatial maps

6.5 General direction verbs

6.5.1 Exophoric general direction verbs

6.5.2 COME and LEAVE

6.6 Summary and discussion

7 Person reference in Kata Kolok

7.1 Overview

7.2 Pronominal pointing to people in sign languages

7.3 Absolute pointing for person reference

7.4 Anaphoric functions of list buoys

7.5 Role shift and pointing

7.6 Summary and discussion

8 Temporal inference in Kata Kolok discourse

8.1 Overview

8.2 Data collection and analysis

8.3 The celestial timeline

8.4 Ambiguity of time adverbial constructions

8.4.1 Time units

8.4.2 PIDAN: the non-present

8.5 Temporal inference

8.5.1 No connectives

8.5.2 Temporal inference and the completive aspect marker

8.5.3 Temporal inference and shared background knowledge

8.5.4 Temporal inference and the modal verb wANT

8.6 Summary and discussion 
Part IV: Sign-spatiality and the dominance of the absolute Frame of Reference in Kata Kolok

9 Frames of Reference in sign languages

9.1 Overview

9.2 Frames of Reference

9.3 Anchoring of the Frames of Reference

9.3.1 Constancy under Rotation

9.3.2 Landmark-based expressions

9.3.3 Landmarks in the Balinese language and culture

9.4 Geocentric Hypothesis

9.5 Sign-spatial significance

9.5.1 The Intrinsic FoR: intrinsic features of the hands

9.5.2 The Relative FoR: viewpoint

9.5.3 The Absolute FoR: absolute directions

9.6 The Rotation Paradigm

9.7 Summary and discussion

10 Simultaneous classifier constructions in narratives

10.1 Overview

10.2 Data collection

10.3 Frames of Reference in Kata Kolok narratives

10.3.1 Absolute sign-spatiality in Kata Kolok narratives

10.3.2 Relative sign-spatiality in a Kata Kolok narrative

10.4 Summary and discussion

11 The Nijmegen Space Games

11.1 Overview

11.2 Man and Tree Game

11.2.1 Methodology Man and Tree Game

11.2.2 Director's reconstructed scene after $90^{\circ}$ rotation (Phase 2)

11.2.3 Directors' descriptions of spatial arrays (Phase 3)

11.2.4 Reconstruction by the matcher (Phase 4)

11.3 Animals in a Row task

11.4 Summary and discussion

Part V: The Kata Kolok pointing system: beyond sign-spatiality

12 Pointing: a brief literature review

12.1 Overview

12.2 Pointing: theoretical issues

12.3 The sign-spatial indeterminacy of pointing

12.4 Grammatical indeterminacy of pointing signs

12.5 Form-meaning mappings of pointing gestures and pointing signs

12.6 Previous work on Kata Kolok pointing

12.7 Summary and discussion

13 Corpus analysis of spontaneous pointing signs

13.1 Overview

13.2 Data collection: spontaneous Kata Kolok pointing signs

13.3 Coding of pointing signs

13.4 General outcomes

13.5 Summary and discussion 
14 Functions and forms of Kata Kolok pointing

14.1 Overview

14.2 Indicating locations

14.2.1 Formal marking of locative pointing signs

14.2.2 Absence of true toponyms

14.3 Indicating people

14.3.1 Ambiguity between place indication and person reference

14.3.2 The grammatical category of first person

14.3.3 Eye-gaze and the grammatical category of person

14.4 Indicating time

14.4.1 Celestial pointing

14.4.2 Cues for celestial pointing

14.5 Lexicalised pointing

14.5.1 Body parts

14.5.2 Pointing for colour

14.5.3 Lexicalisation of pointing signs

14.6 Summary and discussion

15 The semiotic ecologies of pointing

15.1 Overview

15.2 Main findings regarding the Kata Kolok pointing system

15.3 The incremental construction of meaning in Kata Kolok pointing signs

15.4 The grammatical status of Kata Kolok pointing signs

15.5 The grammaticalisation of Kata Kolok pointing signs

15.5.1 Morphemisation

15.5.2 Syntactic integration

15.6 Comparing pointing gestures and pointing signs

Part VI: Conclusion

16 Cross-linguistic conventionalisation of sign-spatiality

16.1 Sign-spatiality in Kata Kolok

16.2 Sources of variation in sign-spatiality

16.3 Future research

16.3.1 Frames of reference and the nature of spatial iconicity

16.3.2 The comparative acquisition of sign-spatial structures

16.3.3 Non-linear conception of time

16.3.4 Grammatical description of Kata Kolok

16.3.5 The scope of generalisations

16.4 Concluding remarks

\section{Author's address}

Connie de Vos

Max Planck Institute for Psycholinguistics

Language and Cognition department

P.O. Box 310

6500 AH Nijmegen

The Netherlands

Connie.deVos@mpi.nl 